\title{
Sustained caloric restriction in health
}

In humans, there are few randomized controlled clinical trials investigating the effects of caloric restriction in health and ageing. Now, Leanne Redman and her team report the results of a 2-year clinical trial that investigated the effect of caloric restriction in healthy humans. The authors found that caloric restriction of $\sim 15 \%$ over 2 years resulted in an average weight loss of $8.7 \mathrm{~kg}$.

In their study, the researchers recruited 53 healthy adults and assigned 34 to the caloric restriction group and 19 to the control group. The caloric restriction group was given a specific diet regime and the control group continued their current diets. During the 2-year study, the team measured the metabolic rate of the participants by various means, including the sleeping metabolic rate (the most robust measurement of human metabolism).

The total body weight of participants in the caloric restriction group was reduced on average by $8.7 \mathrm{~kg}$ ( $70 \%$ of which was from body fat), while the control group gained on average $1.8 \mathrm{~kg}$. In addition, the sleeping metabolic rate of participants in the caloric restriction group fell by $\sim 10 \%$, which was beyond what was expected based on the amount of weight loss observed. The authors also reported that participants in the caloric restriction group had reductions in levels of reactive oxygen species and decreases in thyroid activity (reduced levels of $\mathrm{T}_{3}$ and $\mathrm{T}_{4}$ ), which is a biomarker of decreased ageing.

"The ultimate study into effects of caloric restriction would be one where participants eat a

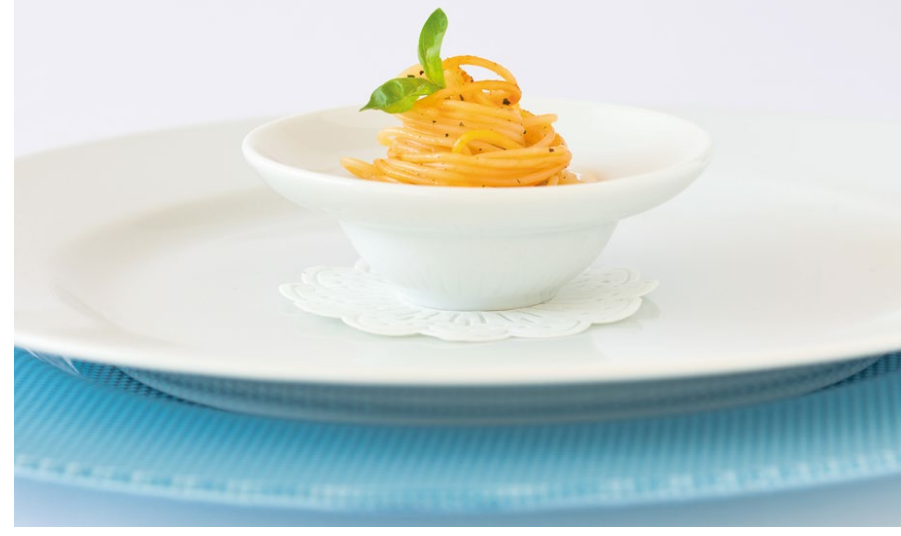

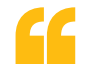

caloric

restriction of

$\sim 15 \%$ over

2 years resulted

in an average

weight loss of

$8.7 \mathrm{~kg}$

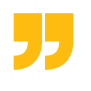

calorie-restricted diet from early adulthood for the remainder of their lives," concludes Redman. "Such a study is the only way to truly answer the question of whether caloric restriction extends lifespan and improves healthspan in people."

Ivone Leong

ORIGINAL ARTICLE Redman, L. M. et al. Metabolic slowing and reduced oxidative damage with sustained caloric restriction support the rate of living and oxidative damage theories of aging. Cell Metab. 27, 1-11 (2018)

\section{Exercise affects gut microbiota and bone}

New research shows that the beneficial effects of exercise on bone are correlated with changes in the composition of the gut microbiota.

"We know that exercise is good for bone and also the gut," explains lead author Laura McCabe (Michigan State University, USA). “So, we were interested in understanding how exercise influences the effects of a high-fat diet on bone and to see if there were links to microbiota composition." The researchers fed male $\mathrm{C} 57 \mathrm{BL} / 6$ mice a low-fat diet ( $10 \%$ of calories from fat) or a high-fat diet ( $60 \%$ of calories from fat) and kept them under sedentary or voluntary exercise conditions for 14 weeks.

A high-fat diet has previously been reported to result in reduced trabecular bone volume in the tibia and vertebrae and increased marrow adiposity. McCabe and her colleagues replicated this finding in male mice, but also confirmed that these effects were ameliorated by exercise. The investigators also found that exercise prevented the adverse effects of a high-fat diet on the gut microbiota, resulting in a healthy gut microbiota composition. Interestingly, the exercise-induced changes in the gut microbiota correlated with changes in bone health parameters. "Pearson's correlation analyses suggest several bacterial family members present in exercised mice correlate with the positive changes to bone," adds McCabe. Microbiome analyses showed that exercise reduced the Firmicutes to Bacteroidetes ratio; furthermore, this ratio was negatively correlated with bone volume. While McCabe and co-workers recognize that further work is needed, they suggest that the changes exercise induced in the gut microbiota could be a novel mechanism via which exercise prevents the negative effects of a high-fat diet on bone.

The researchers are now working on identifying the mechanisms underlying the associations between exercise-induced changes in the gut microbiota and bone health. "We want to know how the microbiota communicate with bone and how we can manipulate the microbiota to prevent bone loss, perhaps by reproducing the microbiota associated with exercise," concludes McCabe.

Claire Greenhill

ORIGINAL ARTICLE McCabe, L. R. et al. Exercise prevents high fat diet-induced bone loss, marrow adiposity and dysbiosis in male mice. Bone https:// doi.org/10.1016/j.bone.2018.03.024 (2018) 